\title{
FACTORES QUE AFECTAN LA ATRACCIÓN DE PROROPS NASUTA WATERSTON (HYMENOPTERA: BATHYLIDAE) A LOS OLORES EMITIDOS POR LOS DESECHOS DE SU HUÉSPED, LA BROCA DEL CAFÉ
}

\author{
Marta Aremi LóPez-Rodríguez ${ }^{1}$, Pilar ChIU-Alvarado ${ }^{1,2}$ \\ y Julio C. RoJAs 1
}

${ }^{1}$ El Colegio de la Frontera Sur, Departamento de Entomología Tropical, Carretera Antiguo Aeropuerto km 2.5, Apdo. Postal 36, CP 30700, Tapachula, Chiapas, MéXICO 2Oxford University, Department of Zoology, South Parks Road, OX1 3PS, Oxford, UK jrojas@ecosur.mx

López-Rodríguez, M. A., P. Chiu-Alvarado y J. C, Rojas. 2009. Factores que afectan la atracción de Prorops nasuta Waterston (Hymenoptera: Bathylidae) a los olores emitidos por los desechos de su huésped, la broca del café. Acta Zool. Mex (n. s.) 25(1): 49-60.

RESUMEN. Prorops nasuta Waterston (Hymenoptera: Bathylidae) es un ectoparásito que se alimenta de todos los estados inmaduros de la broca del café, Hypothenemus hampei Ferrari (Coleoptera: Curculionidae: Scotylinae) y que se reproduce depositando sus huevecillos en la parte ventral de las larvas maduras y pupas de su huésped. Estudios previos han demostrado que este parasitoide es atraído a los olores provenientes de los desechos alimenticios y fecales de su huésped. Este estudio investigó el efecto de algunos factores tales como edad, alimentación, sexo y experiencia de oviposición en la respuesta del parasitoide $P$. nasuta a los olores provenientes de los desechos de su huésped. Los bioensayos se realizaron en un olfactómetro tipo "Y". Los resultados muestran que las hembras de $P$. nasuta $\sin$ alimentar y alimentadas con miel-agua prefirieron significativamente los olores provenientes de los desechos que al control. En contraste, las hembras alimentadas con inmaduros no mostraron ninguna preferencia por los olores de los desechos o por el control. Los resultados muestran que la edad de las hembras no influyó en su respuesta a los olores de los desechos de su huésped. Los machos de $P$. nasuta no fueron atraídos a los olores provenientes de los desechos. La previa experiencia de las hembras no influyó en su respuesta a los olores de los desechos.

Palabras clave: Control biológico, broca del café, Prorops nasuta, localización del huésped, cairomonas.

López-Rodríguez, M. A., P. Chiu-Alvarado \& J. C, Rojas. 2009. Factors affecting the attraction of Prorops nasuta Waterston (Hymenoptera: Bathylidae) to odors emitted by dust/frass of its host, the coffee berry borer. Acta Zool. Mex (n. s.) 25(1): 49-60

ABSTRACT. The parasitoid Prorops nasuta Waterston (Hymenoptera: Bathylidae) is a wasp that feeds upon and parasitizes the coffee berry borer, Hypothenemus hampei (Ferrari) (Coleoptera: Curculionidae: Scolytinae), which is the most important pest of coffee (Coffea spp.) in the world. Previous studies have shown that $P$. nasuta females are attracted to the odors emitted by dust/frass of $H$. hampei. In this study, the effect of age, feeding status, sex and previous experience of $P$. nasuta on 
female attraction to the dust/frass odors was investigated in the laboratory using a Y-tube olfactometer. Females without food and those fed with a solution of honey-water (1: 1) significantly preferred dust/frass odors over clean air. In contrast, females fed with $H$. hampei immature stages did not show any preference for dust/frass odors or clean air. Female age did not influence the attraction of $P$. nasuta to dust/frass odors. $P$. nasuta males were not attracted to dust/frass odors. Both naïve and experienced females were similarly attracted to dust/frass odors, suggesting that the response of $P$. nasuta to dust/frass odors is innate.

Key words: Biological control, coffee berry borer, Prorops nasuta, host location, kairomones.

\section{INTRODUCCIÓN}

Una de las plagas clave del cultivo del café es la broca, Hypothenemus hampei Ferrari (Coleoptera: Curculionidae, Scolytinae), ya que ocasiona daños directos sobre el grano (Baker 1999). El daño es ocasionado por las larvas y adultos que hacen galerías irregulares en el endospermo del fruto (Le Pelley 1968). Este escolítido fue introducido a México a finales de los años 70's del siglo pasado (Baker 1984) y desde entonces diversas medidas de control han sido evaluadas para disminuir sus poblaciones en el campo. Como parte de este esfuerzo, en 1988 se introdujeron desde África a México dos avispitas, Cephalonomia stephanoderis Betrem y Prorops nasuta Waterston (Hymenoptera: Bathylidae), para ser usadas como agentes de control contra H. hampei (Barrera et al. 1990).

Las hembras de $P$. nasuta depredan y parasitan a la broca del café. La biología de esta especie ha sido estudiada por diversos autores (Hargreaves 1935, Abraham et al. 1990, Murphy \& Moore 1990, Infante et al. 2005). Las hembras se alimentan de todos los estados biológicos de la broca, aunque preferencialmente consumen huevecillos, larvas pequeñas y adultos de $H$. hampei durante el periodo de preoviposición. Las hembras de P. nasuta ponen sus huevecillos ventralmente sobre las larvas maduras y pupas de su huésped. Las hembras generalmente solo usan un fruto de café infestado con $H$. hampei, en donde permanecen durante toda su vida. La larva de $P$. nasuta vive en exoparasitismo alimentándose de la hemolinfa de su huésped. El tiempo de desarrollo (huevecillo a adulto) para machos y hembras es aproximadamente de 28 y 30 días, respectivamente (Infante et al. 2005). Los apareamientos ocurren entre hermanos dentro del fruto de café después de lo cual la hembra fertilizada deja el fruto para buscar frutos infestados con H. hampei.

En su actividad diaria, un parasitoide hembra encara el problema no nada más de localizar su alimento sino también tiene que localizar huéspedes en donde depositar sus huevecillos. Es ampliamente aceptado que la eficiencia de un agente de control biológico está correlacionada con su comportamiento de localización de su huésped (Vinson 1998). Durante el proceso de localización de huésped, los parasitoides usan estímulos químicos y físicos provenientes de las plantas de sus huéspedes, de los huéspedes mismos y de los productos de desechos de los huéspedes (Vet \& Dicke 1992). En el caso de P. nasuta se ha encontrado que las hembras son atraídas a los 
frutos infestados por $H$. hampei, y la atracción a esos frutos infestados es debida a los olores emitidos por los inmaduros y los desechos alimenticios y fecales de la broca del café (Chiu-Alvarado et al. 2006, 2007, 2009). Sin embargo, para tener un completo entendimiento del comportamiento de localización de huésped por $P$. nasuta varios otros aspectos necesitan ser investigados. Por ejemplo, se desconoce cuales factores, internos y externos, pueden influir la atracción de $P$. nasuta a los volátiles emitidos por los inmaduros y los desechos de la broca. El estado fisiológico, experiencias previas y condiciones abióticas influyen en la respuesta de los enemigos naturales a los infoquímicos (Lewis et al. 1990, Papaj \& Lewis 1993).

Este estudio fue realizado para investigar el efecto de la edad, la alimentación, el sexo y la experiencia de oviposición de $P$. nasuta en su respuesta a los olores provenientes de los desechos de su huésped.

\section{MATERIAL YMÉTODOS}

Material biológico. Los parasitoides fueron criados siguiendo la técnica y condiciones descritas por Infante et al. (1995). Para la cría del parasitoide fueron utilizados recipientes de plástico (20 $\mathrm{cm}$ de diámetro $\mathrm{x} 8 \mathrm{~cm}$ de altura), en donde se colocaron los frutos de café infestados por la broca. Los frutos seleccionados previamente fueron colocados formando una sola capa dentro de cada recipiente para evitar que hubiera demasiada humedad que pudiera afectar los cultivos. Sólo fueron utilizados aquellos en donde se observó que el polvo de la perforación hecha por la broca era de una coloración oscura. Esto indica que la población de los estados inmaduros dentro del fruto es la adecuada para la reproducción de los parasitoides. Posteriormente fueron introducidas 10 hembras de $P$. nasuta por cada recipiente. Los recipientes fueron revisados después de 20 a 25 días de haber sido introducido los parasitoides para la recuperación de los individuos emergidos. Los parasitoides de $P$. nasuta fueron colectados usando un aspirador eléctrico para depositarlos en un recipiente pequeño de plástico con tapadera. Los recipientes con los parasitoides fueron llevados al laboratorio donde se realizaron los bioensayos para aclimatarlos a las condiciones ambientales de dicho laboratorio.

Los inmaduros (huevo, larva, pre-pupa y pupa) de la broca usados para alimentar a los parasitoides en uno de los experimentos fueron obtenidos de frutos de café infestados por la broca. Los frutos fueron disecados cuidadosamente con una navaja bajo un microscopio, extrayendo los inmaduros con un pincel y depositándolos en una caja de Petri de vidrio.

Los desechos alimenticios y fecales usados como fuente de atracción en los experimentos fueron colectados de los cultivos de broca conservados en el laboratorio. El material de cada recipiente conteniendo los cultivos fue tamizado para separar los desechos de los frutos y eliminar basura. Los desechos fueron depositados en un recipiente pequeño y tapado herméticamente hasta su posterior uso. 
López-Rodríguez et al. Factores que afectan localización de huésped por P. nasuta

Bioensayos. Los bioensayos fueron realizados en un olfatómetro tipo "Y", descrito en detalle por Vander Meer et al. (1988). El olfatómetro estuvo hecho de dos juntas de vidrio de 20/40, unidas a los brazos del tubo de $5 \mathrm{~cm}$ en forma de "Y". Los desechos a evaluar fueron colocados al final de las juntas de vidrio. Los olores son transportados por una corriente de aire previamente purificado con carbón activado. Se utilizó un flujo de aire de $0.2 \mathrm{~L} / \mathrm{min}$ controlado por un par de flujómetros. La técnica del bioensayo fue descrita en detalle por Rojas et al. (2006). Brevemente, los parasitoides fueron colocados individualmente en la entrada del olfatómetro. El bioensayo terminó una vez que los parasitoides habían llegado al final de alguno de los dos brazos del olfatómetro, o que no habían salido del tubo de liberación después de 5 minutos de observación. Los insectos que no respondieron ni a los desechos ni al control fueron considerados como neutrales. Una muestra de $30 \mathrm{mg}$ de desechos fue colocada en un tubo de plástico $(1.4 \mathrm{~cm}$ de largo x $0.7 \mathrm{~cm}$ de diámetro) con ambas entradas tapadas con tela organdí. Este tubo conteniendo los desechos fue colocado en uno de los dos brazos del olfatómetro, en el otro brazo fue colocado un tubo de plástico vacío que fue utilizado como control. La posición de los tubos fue rotada después de cada repetición y después de 5 repeticiones, el olfatómetro fue lavado con agua destilada, jabón neutro y acetona, secado a $100{ }^{\circ} \mathrm{C}$ por $2 \mathrm{hrs}$ y enfriado por $10 \mathrm{~min}$. Los bioensayos fueron realizados a una temperatura de $22 \pm$ $1{ }^{\circ} \mathrm{C}$, humedad relativa de $50 \pm 8 \%$ y una iluminación de 1690 lux provista por 4 lámparas fluorescentes colocadas arriba del olfatómetro.

Efecto de alimentación y edad en hembras $P$. nasuta sobre la respuesta a los olores provenientes de los desechos. Para la realización de este experimento, hembras de $P$. nasuta recién emergidas (1-3 h de edad) fueron separadas en dos grupos de 300 individuos cada uno. De cada grupo, 100 hembras fueron alimentadas con inmaduros de broca ( 5 huevos, 5 larvas, 5 pre-pupas, y 5 pupas), 100 fueron alimentadas con miel diluida en agua (3:1) y a 100 hembras no se le ofreció ningún alimento. El primer grupo de hembras fue evaluado cuando tenían 1-2 días de edad, mientras que el segundo grupo fue bioensayado cuando tenían 4-6 días de edad. En total fueron realizadas 60 repeticiones por grupo, cada repetición consistió de 5 hembras.

Efecto del sexo de $P$. nasuta sobre la respuesta a los olores provenientes de los desechos. Para evaluar el efecto del sexo de P. nasuta sobre su respuesta a los desechos fueron utilizados 100 hembras y 100 machos de 1-2 días de edad. Los parasitoides fueron separados por sexo por su tamaño, las hembras son más grandes que los machos. En total fueron realizadas 20 repeticiones por sexo, cada repetición consistió de 5 individuos.

Efecto de la experiencia de oviposición de $P$. nasuta sobre la respuesta a los olores provenientes de los desechos. Para evaluar el efecto de la experiencia de oviposición de $P$. nasuta sobre la respuesta a los desechos fueron utilizadas 100 hembras de 17 días de edad que habían ovipositado y 70 hembras de 1-2 días de edad 
criadas in vitro que no habían ovipositado. Los parasitoides de 17 días fueron tomados disecando frutos de café tomados del cultivo de la cría de parasitoides. Para el tratamiento con experiencia se hicieron 20 repeticiones, mientras que para el tratamiento sin experiencia se hicieron 14 repeticiones, cada repetición consistió de 5 hembras.

Para criar a los parasitoides in vitro primeramente fue necesario disecar frutos de café infestados por la broca expuesta a los parasitoides. Posteriormente, las pre-pupas y pupas de la broca parasitadas por las larvas de $P$. nasuta fueron colocadas individualmente en cajas pequeñas del plástico $(2 \times 2 \times 3 \mathrm{~cm})$. Una pre-pupa o pupa de $H$. hampei es suficiente para que el parasitoide termine su ciclo de vida (Infante et al. 2005). Las cajas con los huéspedes parasitados fueron mantenidas en una cámara climática (Percival, EUA) a $25 \pm 1{ }^{\circ} \mathrm{C}$ y $60 \pm 10 \%$ humedad relativa hasta la emergencia del parasitoide adulto.

Análisis estadístico. Los resultados obtenidos sobre el efecto de la alimentación y edad en la respuesta de $P$. nasuta a los olores provenientes de los desechos fueron examinados mediante la aplicación de un modelo Log-Lineal con una estructura de error de Poisson. El estadístico utilizado fue el denominado cociente de verosimilitud. El modelo estadístico fue ajustado usando el programa S-Plus 7.0 para Windows (Insightful Corporation, EUA). Los datos de influencia del sexo y experiencia de oviposición fueron analizados por la prueba de G con corrección de Williams (Sokal \& Rohlf 1995).

\section{RESULTADOS}

Efecto de la alimentación y la edad en hembras $P$. nasuta sobre la respuesta a los olores provenientes de los desechos. Los resultados del análisis estadístico de los datos se muestran en el Cuadro 1 . Se aprecia que las variables alimento y edad no fueron significativas, pero sí el tipo de respuesta (a los desechos, al control o neutral). También se observó que las interacciones Alimento * Tipo de Respuesta, y Edad * Tipo de Respuesta fueron significativas.

La interacción entre el tipo de respuesta de las hembras de P. nasuta y la alimentación se muestran en la Fig. 1. Los resultados muestran que las hembras de $P$. nasuta sin alimentar y alimentadas con miel-agua prefirieron significativamente los olores provenientes de los desechos al aire limpio, y solo un pequeño número de insectos fueron neutrales. En contraste, las hembras alimentadas con inmaduros no mostraron ninguna preferencia por los desechos o el control, y un número importante de los parasitoides fueron neutrales. 
López-Rodríguez et al. Factores que afectan localización de huésped por P. nasuta

\section{Cuadro 1}

Resultados del modelo Log-Lineal. Nivel de significancia real para los datos de la respuesta de hembras de Porops nasuta de diferente estatus alimenticio y edad a los volátiles de los desechos alimenticios y fecales de su huésped.

\begin{tabular}{clccc} 
Modelo & \multicolumn{1}{c}{ Variables } & $\mathbf{G}^{\mathbf{2}}$ & g.l. & P \\
\hline Log-lineal & Alimento (A) & 0.76000 & 2 & 0.683 \\
& Edad (B) & 0.10000 & 1 & 0.752 \\
& Tipo de Respuesta (C) & 93.74000 & 2 & $<0.001$ \\
& Alimento*Tipo de Respuesta & 26.34000 & 4 & $<0.001$ \\
& Edad*Tipo de Respuesta & 8.86000 & 2 & 0.011 \\
\hline
\end{tabular}

Prueba $\mathrm{G}^{2} \mathrm{del}$

Modelo: A, B, C, AC, BC

$\begin{array}{lll}11.35000 \quad 6 & 0.0781\end{array}$

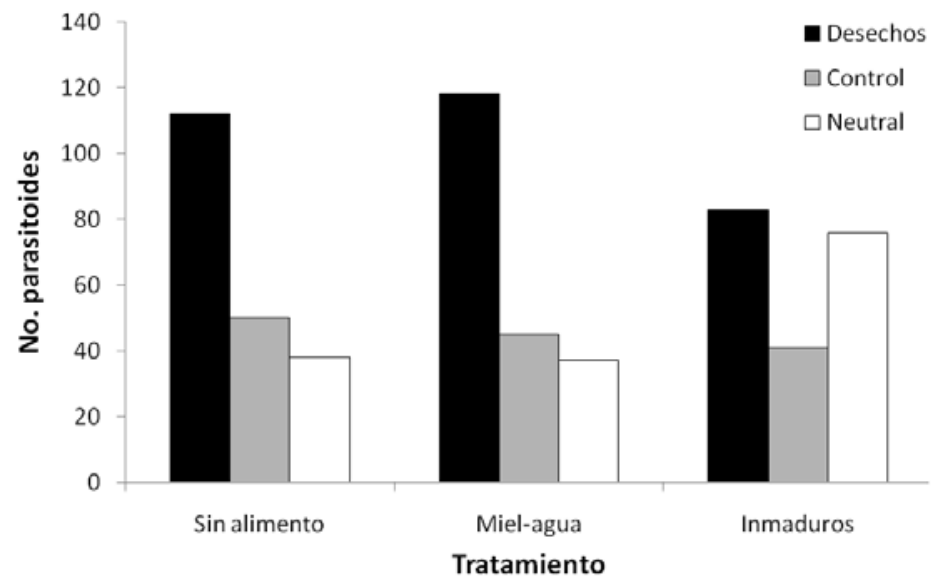

Figura 1. Interacción entre el tipo de alimento (miel-agua, inmaduros y sin alimentol) y el tipo de respuesta (desechos, control, neutral) de hembras de Porops nasuta. DAF, desechos alimenticios y fecales. Las hembras que no respondieron a los olores provenientes de los desechos o al control fueron consideradas neutrales.

La interacción entre el tipo de respuesta de las hembras de $P$. nasuta y la edad se muestran en la Fig. 2. La interacción se debe a los cambios en el numero de respuestas control y neutral en los tratamientos de edad. Los resultados muestran que las hembras de 1-2 o 4-6 días de edad fueron atraídas en mayor número a los desechos que aquellas que respondieron al control o fueron neutrales. 


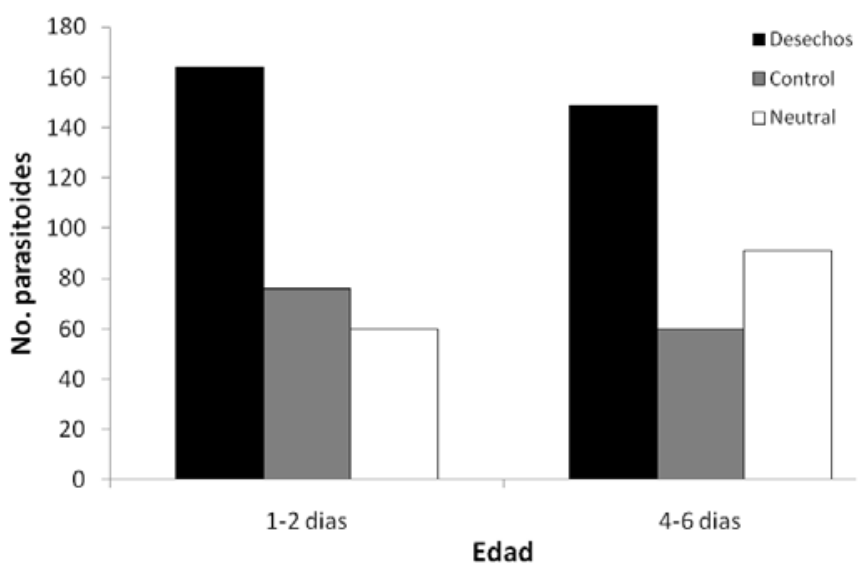

Figura 2. Interacción entre la edad (1-2 días y 4-6 días) y tipo de respuesta (desechos, control, neutral) en hembras de Porops nasuta. DAF, desechos alimenticios y fecales. Las hembras que no respondieron a los olores provenientes de los desechos o al control fueron consideradas neutrales.

Efecto del sexo de $P$. nasuta sobre la respuesta a los olores provenientes de los desechos. Las hembras de $P$. nasuta significativamente prefirieron los olores provenientes de los desechos que al control $(\mathrm{G}=9.94$, g.l. $=1, \mathrm{P}=0.001)$. En contraste, los machos no mostraron ninguna preferencia por los desechos o el control $(\mathrm{G}=0.018$, g.l. $=1, \mathrm{P}=0.8932$, Fig. 3).

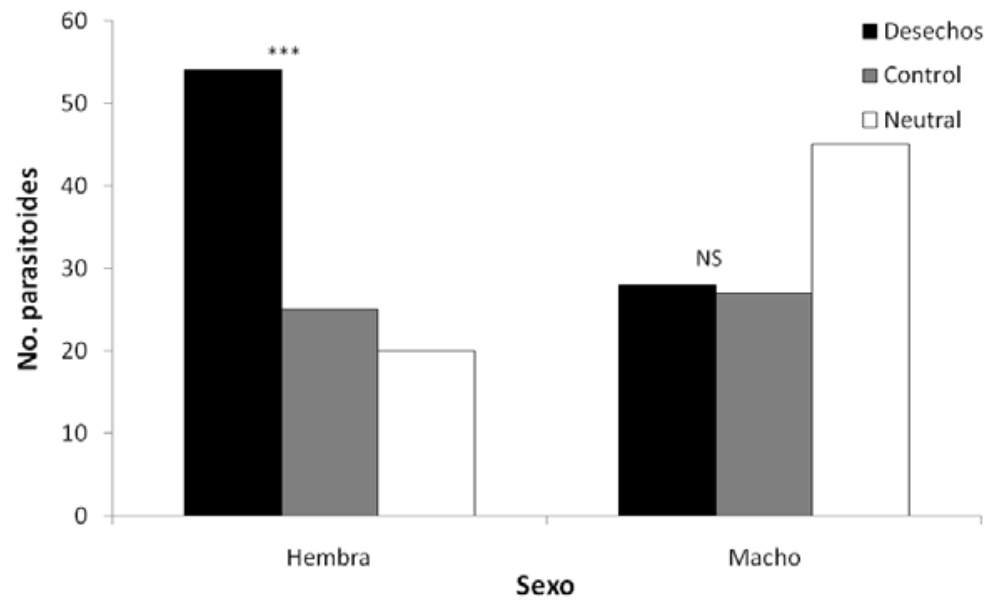

Figura 3. Respuesta de hembras y machos de Prorops nasuta mostrada a los desechos alimenticios y fecales $(\mathrm{DAF})$ o el control. El * indica diferencia significativa $(\mathrm{P}<0.05)$, NS indica diferencia no significativa $(\mathrm{P}>0.05)$. Las hembras que no respondieron a los olores provenientes de los desechos o al control fueron consideradas neutrales. 
Efecto de la experiencia de oviposición de $\boldsymbol{P}$. nasuta sobre la respuesta a los olores provenientes de los desechos. La respuesta a los olores de los desechos por las hembras de $P$. nasuta con experiencia de oviposición y sin experiencia fue significativamente mayor que al control (Fig. 4). Al comparar el numero de hembras con experiencia que respondieron a los desechos con respecto a la respuesta de las hembras sin experiencia, no se encontró diferencias ya que en ambos grupos de hembras la respuesta a los desechos fue $\operatorname{similar}(\mathrm{G}=2.13$, g.l. $=1, \mathrm{P}=0.14)$.

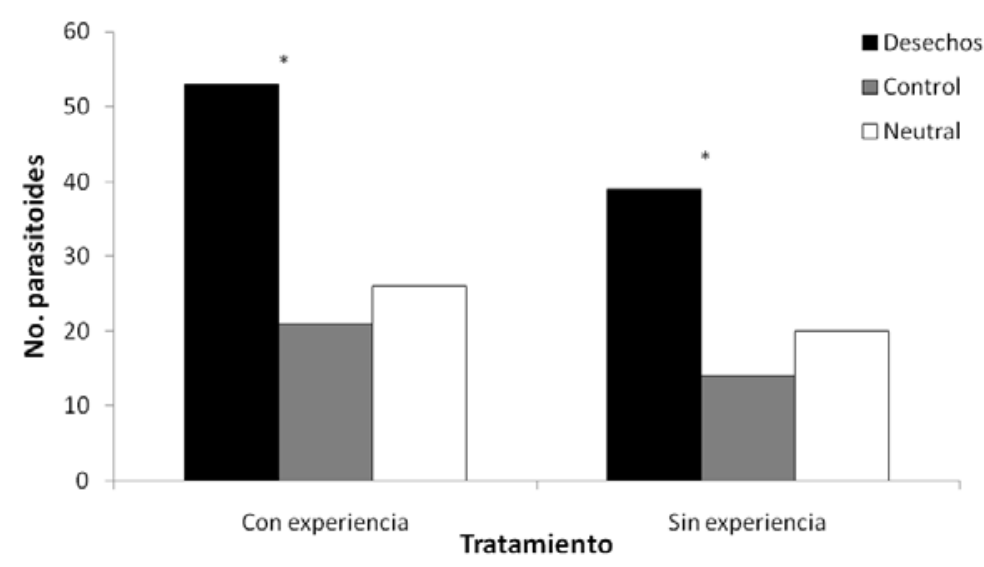

Figura 4. Respuesta de Prorops nasuta con y sin experiencia en oviposición a los desechos alimenticios y fecales (DAF) o el control. * indica diferencia significativa $(\mathrm{P}<0.05)$. Las hembras que no respondieron a los desechos olores provenientes de los o al control fueron consideradas neutrales.

\section{DISCUSIÓN}

Varios estudios han mostrado que la respuesta de los parasitoides a los estímulos usados durante el proceso de localización de huésped puede ser influenciado por diversos factores internos y externos tales como la edad, la alimentación, la temperatura y la humedad relativa, entre otros (Lewis et al. 1998). Los resultados del presente estudio demuestran que las hembras $P$. nasuta son atraídas a los olores de los desechos sólo cuando no están alimentadas o cuando son alimentadas con mielagua, pero no cuando son alimentadas con inmaduros. Típicamente una hembra de $P$. nasuta entra a una cereza infestada por la broca del café e inicialmente se alimenta de los estados inmaduros del huésped. Después varios días de haberse alimentado empieza a ovipositar sobre las larvas maduras y prepupas de H. hampei (Infante et al. 2005). Los resultados de este estudio sugieren que las hembras de P. nasuta utilizan los olores provenientes de los desechos como un estímulo primeramente para 
localizar huéspedes con fines de alimentación más que de oviposición. Debido a que los desechos están íntimamente asociados con los inmaduros de la broca, entonces los olores provenientes de los desechos se convierten en una señal altamente confiable para los parasitoides de la presencia de su huésped.

Los resultados también sugieren que una vez que los insectos se han alimentado su estado fisiológico cambia y por lo tanto ya no son atraídos a los volátiles de los desechos de su huésped. Sin embargo, se encontró que la edad no afectó la respuesta de las hembras a los olores de los desechos de su huésped. De hecho, hembras de 17 días de edad fueron atraídas a los desechos en el experimento de experiencia confirmando que la respuesta de las hembras a los olores de su huésped no es afectada por la edad, aunque si por la alimentación. En este sentido, Takasu \& Lewis (1993) encontraron que la privación del alimento y la disponibilidad de éste son factores importantes que afectan el comportamiento de localización del huésped de Microplitis croceipes (Cresson), (Hymenoptera: Braconidae) un parasitoide de Helicoverpa zea Boddie y Heliothis virescens (Fabricius) (Lepidoptera: Noctuidae). Así, la privación del alimento afectó la motivación de este parasitoide para buscar a H. zea, lo cual tiene consecuencias directas en su eficacia de parasitización (Stapel et al. 1997). La privación del alimento es un factor que influyó sobre las decisiones de forrajeo de Bathyplectes curculionis (Thomson) (Hymenoptera: Ichneumonidae), un parasitoide de la larva del picudo de la alfalfa, Hypera postica (Gyllenhal) (Coleoptera: Curculionidae) (Jacob \& Evans 2001); y de Cotesia rubecula (Marshall) (Hymeoptera: Braconidae) parasitoide de Pieris rapae (L.) (Lepidoptera: Pieridae)(Wäckers 1994). En este último caso se encontró que los parasitoides prefirieron los olores provenientes de flores cuando estaban hambrientos, pero una vez que se han alimentado con miel cambiaron su preferencia por los olores provenientes de plantas infestadas por su huésped (Wackers 1994). La previa alimentación de Diadegma insulare (Cresson) (Hymenoptera: Ichneumonidae) con sacarosa no afectó su tasa de parasitización, pero las hembras con esta dieta encontraron más rápidamente a su huésped Plutella xylostella (L.) (Lepidoptera: Plutellidae), ya que éstas no invirtieron más tiempo en la búsqueda de alimento (Lee \& Heimpel 2007).

En el presente estudio se encontró que la respuesta de $P$. nasuta a los olores de los desechos depende del sexo. La hembras de $P$. nasuta son las únicas que fueron atraídas a los olores provenientes de los desechos alimenticios y fecales de su huésped. Este resultado está de acuerdo con la biología de $P$. nasuta ya que los machos no se alimentan de los huéspedes y se conoce que al emerger se aparean con sus hermanas dentro del fruto del café (Infante et al. 2005). En contraste, las hembras son las encargadas de la reproducción, al emerger tienen que salir a buscar nuevas brocas para alimentarse y para fines de oviposición (Bourbón 2004). En contraste con los resultados del presente trabajo, Takasu et al. (2007) encontraron que los 
machos de M. croceipes pueden responder a los olores emitidos por el huésped tan bien como las hembras. Usando su capacidad de aprendizaje y su habilidad para la detección a olores, los machos de los $M$. croceipes pueden buscar la fuente del alimento tan eficientemente como hembras bajo condiciones naturales aunque la razón de este tipo de comportamiento no es clara.

Los resultados del presente estudio mostraron que las hembras $P$. nasuta tienen respuestas innatas a los olores provenientes del huésped y los datos obtenidos sugieren que no aprenden. Estos resultados están de acuerdo con la hipótesis que predice que a una mayor especialización alimentaría existe una menor necesidad de aprender (Steidle \& van Loom 2003). Es conocido que P. nasuta tiene un limitado número de huéspedes (Barrera et al. 1989) y en el campo sólo se le ha encontrado atacando a H. hampei. En contraste con nuestros resultados, las hembras sin experiencia de Habrobracon hebetor Say (Hymenoptera: Braconidae) no son atraídas a los desechos de su huésped, Ephestia kuehniella Zeller (Lepidoptera: Pyralidae), mientras que aquellas que sí tenían experiencia sí fueron atraídas a los desechos (Parra et al. 1996). El parasitoide Aphytis melinus DeBach (Hymenoptera: Aphelinidae) es atraído a los volátiles emitidos su huésped Aonidiella aurantii (Maskell) (Homoptera: Diaspididae) o al complejo planta-huésped sólo después que ha tenido una experiencia previa con el huésped o con el complejo planta- huésped (Morgan \& Hare 1998). Las hembras de Itoplectis naranyae Ashmead (Hymenoptera: Ichneumonidae) con experiencia previa en oviposición encontraron más rápidamente a su huésped en comparación con las hembras sin experiencia. Además una sola oviposición es suficiente para que las avispas acorten el tiempo requerido para encontrar a un nuevo huésped (Kanako \& Ueno 2005).

En conclusión, la alimentación y el sexo de $P$. nasuta afectaron su respuesta a los olores provenientes de los desechos del huésped. Desde el punto de vista práctico los resultados sugieren que la eficiencia de $P$. nasuta como un agente de control biológico de la broca puede ser afectado por el tipo de alimentación a la que tienen acceso los parasitoides. La respuesta de las hembras de $P$. nasuta a los olores emitidos por los desechos del huésped parece ser innata pero más experimentos son necesarios para establecer claramente que las hembras de $P$. nasuta no tienen la capacidad de aprender los olores asociados a su huésped.

AGRADECIMIENTOS. Se agradece al Dr. Juan Barrera por las facilidades prestadas en los laboratorios de la línea Manejo Integrado de Plagas del ECOSUR, al Q. A. Enrique López por el apoyo en la cría del material biológico usado en los experimentos, y al M. en C. Javier Valle por la asesoría en el análisis estadístico de los datos. Al CONACYT por el financiamiento otorgado a través de un proyecto a Julio C. Rojas (No. 40338-Q, investigación básica SEP-CONACYT) y por una beca a Pilar Chiu Alvarado para realizar sus estudios de doctorado. 


\section{LITERATURA CITADA}

Abraham, Y. J., D. Moore \& G. Godwin. 1990. Rearing and aspects of biology of Cephalonomia stephanoderis and Prorops nasuta (Hymenoptera: Bethylidae) parasitoids of the coffee berry borer, Hypothenemus hampei (Coleoptera: Scolytidae). Bulletin of Entomological Research 80: 121-128.

Baker, P. S. 1984. Some aspects of the behaviour of the coffee berry borer in relation to its control in southern Mexico (Coleoptera, Scolytidae). Folia Entomologica Mexicana 61: 9-24.

Baker, P. S. 1999. La broca del café en Colombia. Informe Final del proyecto MIP para el café DFIDCENICAFE-CABI. Chinchiná, Colombia. 154 pp.

Barrera, J. F., J. Gómez, F. Infante, A. Castillo \& W. De La Rosa. 1989. Biologie de Cephalonomia stephanoderis Betrem (Hymenoptera: Bethylidae) en laboratoire: I. Cycle biologique, capacité d'oviposition et émergence du fruit du caféier. Café, Cacao, Thé 33: 101-108.

Barrera, J. F., J. Valenzuela \& P. S. Baker. 1990. Introducción de dos especies de parasitoides Africanos a México para el control biológico de la broca del cafeto Hypothenemus hampei (Ferrari) (Coleoptera: Scolitydae). Folia Entomológica Mexicana 79: 245-247.

Bourbón, O. M. 2004. El uso de parasitoides Prorops nasuta y Phymastichus coffea para el control de la broca del fruto del cafeto. Boletín informativo. Oficina Regional Los Santos. San Marcos de Tarrazú, Costa Rica. Año 4, Número 1. 6 p.

Chiu-Alvarado, P., A. Virgen \& J. C. Rojas. 2006. Atracción de Prorops nasuta, un parasitoide de la broca del café, a los estímulos olfativos asociados al huésped. Pp. 404-409. En: Estrada Venegas, E. G., J. Romero Nápoles, A. Equihua Martínez, C. Luna León y J. L. Rosas Acevedo (Eds), Entomología Mexicana, Vol. 5, tomo 1, Sociedad Mexicana de Entomología.

Chiu-Alvarado, P., A. Virgen \& J. C. Rojas. 2007. Comportamiento de localización de huésped por los parasitoides de la broca del café. Pp. 121-130. En: Barrera, J. F., A. García, V. Domínguez \& C. Luna (Eds), La broca del café en América tropical: Hallazgos y enfoques. El Colegio de la Frontera Sur. México.

Chiu-Alvarado, P., J. F. Barrera \& J. C. Rojas. 2009. Attraction of Prorops nasuta (Hymenoptera: Bethylidae), a parasitoid of the coffee berry borer (Coleoptera: Curculionidae), to host-associated olfactory cues. Annals of the Entomological Society of America 102: 000-000.

Hargreaves H. 1935. Stephanoderes hampei, Ferr., coffee berry borer in Uganda. East African Agricultural Journal 1:218-224.

Infante, F., J. Mumford \& P. Baker. 2005. Life history of Prorops nasuta, a parasitoid of the coffee berry borer. BioControl 50: 259-270.

Jacob, H. S. \& E. W. Evans. 2001. Influence of food deprivation on foraging decisions of the Parasitoid Bathyplectes curculionis (Hymenoptera: Ichneumonidae) Annals Entomological Society of America 94: 605-611.

Kanako, U. \& T. Ueno. 2005. Effect of wasp size, physiological state, and prior host experience on host-searching behavior in a parasitoid wasp (Hymenoptera: Ichneumonidae). Journal of Ethology 23: 43-49.

Le Pelley, R.H. 1968. Pests of coffee. Longmans Green and Co., London, England.

Lee, J.C. \& G. E. Heimpel. 2007. Sugar feeding reduces short-term activity of a parasitoid wasp. Physiological Entomology 32: 99-103.

Lewis, W. J. L. E. M. Vet., J. H. Tumlinson J. C. van Lenteren \& D. R Papaj. 1990. Variations in parasitoid foraging behavior: essential elements of a sound biological control theory. Environmental Entomology 19: 1183-1193.

Lewis, W. O. Stapel, A Cortesero \& K Takasu. 1998. Understanding how parasitoids balance food and host needs: importance to biological control. Biological Control 11: 175-183.

Morgan, D. J. W. \& J. D. Hare. 1998. Volatile cues used by the parasitoid, Aphitis melinus, for host 
location: California red scale revisited. Entomologia Experimentalis et Applicata. 88: 235-245.

Murphy, S.T. \& D. Moore. 1990. Biological control of the coffee berry borer, Hypothenemus hampei (Ferrari) (Coleoptera: Scolitydae): previous programmes and possibilities for the future. Biocontrol News Information 11: 107-117.

Papaj, R. D \& A. C. Lewis. 1993. Insect learning. Pp 51-79. En: Papaj, D. R. \& A. C. Lewis (Eds). Insect learning: ecological and evolutionary perspectives. Chapman and Hall, New York..

Parra, J. R. P., S. B. Vinson, S. M. Gomes \& F. L. Consoli. 1996. Flight response of Habrobracon hebetor (Say) (Hymenoptera: Braconidae) in a wind tunnel to volatiles associated with infestations of Ephestia kuehniella Zeller (Lepidoptera: Pyralidae). Biological Control 6: 143-150.

Rojas, J. C., A. Castillo \& A. Virgen. 2006. Chemical cues in host location by Phymastichus coffea, a parasitoid of coffee berry borer adults, Hypothenemus hampei. Biological Control 37: 141-147

Steidle, J. L. M. \& J. J. A. Van Loom. 2003. Dietary specialization and infochemical use in carnivorous arthropods: Testing a concept. Entomologia Experimentalis et Applicata. 108: 133-148.

Sokal, R. R. \& F. J. Rohlf. 1995. Biometry. Third edition. W. H. Freeman and company, New York.

Stapel, J. O., A.M. Cortesero, C.M. De Moraes, J.H. Tumlinson \& W. J. Lewis. 1997. Extrafloral nectar, honeydew, and sucrose effect on searching behavior and efficiency of Microplitis croceipes (Hymenoptera: Braconidae) in cotton. Environmental Entomology 26: 617-627.

Takasu, K. \& W. J. Lewis. 1993. Host-and food-foraging of the parasitoid Microplitis criceipes: Learning and physiological state effects. Biological Control 3: 70-74.

Takasu, K., G. R. Rains \& W. J. Lewis. 2007. Comparison of detection ability of learned odors between males and females in the larval parasitoid Microplitis croceipes. Entomologia Experimetalis et Applicata 122: 247-251.

Vander Meer, M. R. K., F. Alvarez \& C. S. Lofgren. 1988. Isolation of the trail recruitment pheromone of Solenopsis invicta. Journal of Chemical Ecology 14: 825-838.

Vet, L. E. M., \& M. Dicke. 1992. Ecology of infochemical use by natural enemies in a tritrophic context. Annual Review of Entomology 37: 141-172.

Vinson, S. B. 1998. The general host selection behavior of parasitoid Hymenoptera and a comparison of initial strategies utilized by larvaphagous and oophagous species. Biological Control 11: 79-96.

Wäckers, F. L. 1994. The effect of food-deprivation on the innate visual and olfactory preference in the parasitoid Cotesia rubecula. Journal of Insect Behavior 40: 641-649.

Recibido: 6 de febrero de 2008

Aceptado: 2 de diciembre de 2008 